\title{
Initial Value Methods for Parabolic Control Problems
}

\section{By Ragnar Winther}

\begin{abstract}
We study iterative methods for parabolic control problems with a Neumann boundary value control and where the observation is the final state. The methods are based on transforming the original control problem (which may have constraints on the control) into an equivalent problem of minimizing a strictly convex functional (no constraints). The methods are semidiscrete in the sense that we assume that parabolic initial value problems can be solved exactly.
\end{abstract}

1. Introduction. The purpose of this paper is to study approximations of certain parabolic control problems. In order to describe these problems, let $\Omega$ be a bounded domain in $\mathbf{R}^{d}$ with sufficiently smooth boundary, $\partial \Omega$, and for a fixed $T>0$ let $Q=(0, T) \times \Omega$ and $\Sigma=(0, T) \times \partial \Omega$.

On the domain $\Omega$ let $L$ denote the second order differential operator

$$
L u=-\sum_{i, j=1}^{d} \frac{\partial}{\partial x_{i}}\left(a_{i, j}(x) \frac{\partial u}{\partial x_{j}}\right)+c(x) u .
$$

We will assume that $a_{i, j}$ and $c$ are sufficiently smooth real-valued functions on $\bar{\Omega}$ and that the operator $L$ is strongly elliptic; i.e., there is a constant $c_{1}>0$ such that

$$
\sum_{i, j=1}^{d} a_{i, j}(x) \xi_{i} \xi_{j} \geqslant c_{1} \sum_{i=1}^{d} \xi_{i}^{2}
$$

for all $x \in \Omega$ and $\xi \in \mathbf{R}^{d}$.

Now let $v_{1} \in L^{2}(\Omega)$ and $f \in L^{2}(Q)$ be given and for any $g \in L^{2}(\Sigma)$, let $u_{g}=$ $u_{g}(t, x)$ denote the unique weak solution of the problem

$$
\begin{array}{ll}
d u / d t+L u=f & \text { on } Q, \\
\partial u / \partial \nu=g & \text { on } \Sigma, \\
u(0, \cdot)=v_{1} & \text { on } \Omega .
\end{array}
$$

Here $\partial / \partial \nu=\sum_{i, j=1}^{d} a_{i, j} n_{i}\left(\partial / \partial x_{j}\right)$, where $n_{i}$ is the $i$ th component of the outward unit normal on $\partial \Omega$.

We shall consider optimal control problems of the form

$$
\min _{g \in K}\left\{\left\|u_{g}(T, \cdot)-v_{2}\right\|_{L^{2}(\Omega)}^{2}+\alpha\|g\|_{L^{2}(\Sigma)}^{2}\right\},
$$

Received November 27, 1978.

AMS (MOS) subject classifications (1970). Primary $49 \mathrm{D05}$. 
where $v_{2} \in L^{2}(\Omega)$ and $\alpha>0$ are given and where $K$ is a closed convex set of $L^{2}(\Sigma)$. In Section 2 we shall consider the case when $K=L^{2}(\Sigma)$, while in Section 4 we consider $K=\left\{g \in L^{2}(\Sigma) \mid g \geqslant 0\right.$ a.e. on $\left.\Sigma\right\}$.

In both cases we shall derive algorithms where approximations of the solution of (1.2) are generated by solving linear initial value problems of the form (1.1). This will be done by showing that (1.2) (in both cases mentioned above) is equivalent to minimizing a strictly convex functional over the entire space $L^{2}(\Omega)$. The minimum of this functional can then be found by standard iterative techniques. In the case $K=$ $L^{2}(\Sigma)$ the associated functional is quadratic, and in Section 3 we study how this functional can be minimized by the conjugate gradient method. We shall particularly show certain superlinear convergence estimates for this method by applying some results recently given by the author in [13].

In order to evaluate the functionals mentioned above we have to solve two initial value problems of the form (1.1). Hence, the approximation of (1.2) is reduced to the well-studied problem of solving problems of the form (1.1) numerically. Such numerical methods will not be considered here. We shall instead assume that problems of the form (1.1) can be solved exactly. However, we mention that a detailed study of fully discrete analogs of the methods considered here was done by the author in [11] and [12] in the case when $K=L^{2}(\Sigma)$, and some numerical examples can be found in [11].

2. Preliminaries. We start with some notation. If $H_{1}$ and $H_{2}$ are two Hilbert spaces then $L\left(H_{1}, H_{2}\right)$ will denote the set of bounded linear maps from $H_{1}$ to $H_{2}$. On the spaces $L^{2}(\Omega)$ and $L^{2}(\partial \Omega)$, respectively, we shall use the notation

$$
(\varphi, \psi)=\int_{\Omega} \varphi \psi d x \quad \text { and } \quad\langle\varphi, \psi\rangle=\int_{\partial \Omega} \varphi \psi d \sigma
$$

for the inner products, and the associated norms will be denoted by $\|\cdot\|$ and $|\cdot|$.

If $p \geqslant 0$, let $H^{p}(\Omega)$ denote the Sobolev space of order $p$ of real-valued functions on $\Omega$ (see for example Lions and Magenes [5]). If $p<0, H^{p}(\Omega)$ is defined to be the dual of $H^{-p}(\Omega)$ with respect to the inner product of $L^{2}(\Omega)$. Furthermore, we let

$$
W(0, T)=\left\{f \mid f \in L^{2}\left(0, T ; H^{1}(\Omega)\right), \frac{d f}{d t} \in L^{2}\left(0, T ; H^{-1}(\Omega)\right)\right\},
$$

where $d / d t$ is taken in the sense of distributions on $(0, T)$ with values in $H^{1}(\Omega)$. We recall from [5] that $W(0, T) \subset C\left(0, T ; L^{2}(\Omega)\right)$.

Let also $B: H^{1}(\Omega) \times H^{1}(\Omega) \rightarrow \mathrm{R}$ denote the bilinear form associated with the operator $L$; i.e.,

$$
B(\varphi, \psi)=\int_{\Omega}\left\{\sum_{i, j=1}^{n} a_{i, j}(x) \frac{\partial \varphi}{\partial x_{i}} \frac{\partial \psi}{\partial x_{j}}+c(x) \varphi \psi\right\} d x .
$$

The problem (1.1) can now be written in the variational form

$$
\left\{\begin{array}{l}
(d u / d t, \varphi)+B(u, \varphi)=(f, \varphi)+\langle g, \varphi\rangle \quad \text { for } \varphi \in H^{1}(\Omega) \\
u(0, \cdot)=v_{1}
\end{array}\right.
$$


From [5] we know that if $v_{1} \in L^{2}(\Omega), g \in L^{2}(\Sigma)$ and $f \in L^{2}(Q)$, then (2.1) has a unique solution in $W(0, T)$.

If $w \in W(0, T)$ is a solution of the "adjoint problem"

$$
\begin{aligned}
(d w / d t, \varphi)+B(\varphi, w) & =0 \quad \text { for } \varphi \in H^{1}(\Omega), \\
w(0, \cdot) & =z,
\end{aligned}
$$

for some $z \in L^{2}(\Omega)$ then we let $E(t) z=w(t, \cdot)$. By using parabolic regularity and certain trace theorems from [5] we then find that the map

$$
\left.z \rightarrow E(\cdot) z\right|_{\Sigma}
$$

is continuous from $L^{2}(\Omega)$ into $L^{2}(\Sigma)$. (In fact, adopting the notation from [5], the map is continuous from $L^{2}(\Omega)$ into $H^{1 / 2,1 / 4}(\Sigma)$.)

3. The Case of No Constraints. Throughout this section we consider the control problem (1.2) only in the case when $K=L^{2}(\Sigma)$. We shall show that this problem is equivalent to minimizing a quadratic functional over $L^{2}(\Omega)$. It is well known that (1.2) has a unique solution. The following characterization of this solution is given by Lions [4].

Lemma 3.1. Let $g \in L^{2}(\Sigma)$ and let $u=u_{g}(t, x)$ be the corresponding solution of (1.1). Then $g$ is an optimal solution of (1.2) if and only if there is $a w \in W(0, T)$ such that

$$
\left\{\begin{array}{l}
(d u / d t, \varphi)+B(u, \varphi)+\alpha^{-1}\langle w, \varphi\rangle=(f, \varphi) \quad \text { for } \varphi \in H^{1}(\Omega) \\
-(d w / d t, \varphi)+B(\varphi, w)=0 \quad \text { for } \varphi \in H^{1}(\Omega) \\
u(0, \cdot)=v_{1}, \quad w(T)=u(T, \cdot)-v_{2}
\end{array}\right.
$$

where $g=-\left.\alpha^{-1} w\right|_{\Sigma}$.

The disadvantage of this characterization of the optimal control is that the system (3.1) is coupled in time. Hence it is expensive to solve numerically. We also observe that, if $w(T)$ were known, then the solution of (3.1) (and hence the solution of (1.2)) could be found by solving two parabolic initial value problems.

The purpose of the rest of this section is to give an alternative characterization of $w(T)$. From this new characterization we can derive methods for approximating $w(T)$ directly. We shall first study a parabolic system where the coupling between the two unknown functions is simpler than in (3.1). For any given $z \in L^{2}(\Omega)$ consider the system

$$
\left\{\begin{array}{l}
(d \hat{u} / d t, \varphi)+B(\hat{u}, \varphi)=\alpha^{-1}\langle\hat{w}, \varphi\rangle \quad \text { for } \varphi \in H^{1}(\Omega) \\
-(d \hat{w} / d t, \varphi)+B(\varphi, \hat{w})=0 \quad \text { for } \varphi \in H^{1}(\Omega) \\
\hat{u}(0, \cdot)=0, \quad \hat{w}(T, \cdot)=z
\end{array}\right.
$$

This system has a unique solution $\hat{u}, \hat{w} \in W(0, T)$. We now define an operator $R$ : $L^{2}(\Omega) \rightarrow L^{2}(\Omega)$ by $R z=\hat{u}(T)$. In fact, by using parabolic regularity and trace theo- 
rems from [5], it follows that $R \in L\left(L^{2}(\Omega) ; H^{1}(\Omega)\right)$; hence Rellich's Lemma implies that $R$ is a compact operator on $L^{2}(\Omega)$. Note also that an evaluation of the operator $R$ requires that two parabolic initial value problems be solved.

Next we wish to show that $R$ is positive semidefinite on $L^{2}(\Omega)$. In order to see this let $z, z^{\prime} \in L^{2}(\Omega)$ and let $\hat{u}, \hat{w}$ and $u^{\prime}, w^{\prime}$, respectively, be the corresponding solutions of (3.2). Then

$$
\left(d \hat{u} / d t, w^{\prime}\right)+B\left(\hat{u}, w^{\prime}\right)=\alpha^{-1}\left\langle\hat{w}, w^{\prime}\right\rangle=\left(d u^{\prime} / d t, \hat{w}\right)+B\left(u^{\prime}, \hat{w}\right)
$$

or

$$
\left(R z, z^{\prime}\right)=\int_{0}^{T} \frac{d}{d t}\left(\hat{u}, w^{\prime}\right) d t=\int_{0}^{T} \frac{d}{d t}\left(u^{\prime}, \hat{w}\right) d t=\left(R z^{\prime}, z\right)
$$

Similarly, we also obtain

$$
(R z, z)=\alpha^{-1} \int_{0}^{T}|\hat{w}|^{2} d t \geqslant 0 .
$$

Hence, we have shown that the operator $R$ defined by (3.2) is a positive semidefinite, compact operator on $L^{2}(\Omega)$.

Now let $u_{0} \in W(0, T)$ be the solution of the initial value problem (1.1) with $g \equiv 0$, and let $b_{0}=u_{0}(T)-v_{2}$. Furthermore, let $u, w$ be the solution of (3.1) and let $z^{*}=w(T)$. Then

$$
z^{*}+v_{2}=u(T)=u_{0}(T)-R z^{*} \quad \text { or } \quad(I+R) z^{*}=b_{0} .
$$

The equation (3.3) can now be used as a starting point for approximating $z^{*}$. In Section 4 we shall show how the conjugate gradient method can be used to find approximations of $z^{*}$. For each iteration one application of $R$ is needed, and hence two parabolic initial value problems must be solved. We also observe that $z^{*}$ minimizes the quadratic functional

$$
\Psi_{0}(z)=1 / 2((I+R) z, z)-\left(b_{0}, z\right) .
$$

Hence, we have shown that the control problem (1.2) (where the control variable is in $L^{2}(\Sigma)$ ) is equivalent to minimizing the quadratic functional $\Psi_{0}$ over $L^{2}(\Omega)$.

4. Application of the Conjugate Gradient Method. In this section we let $(\cdot, \cdot)$ denote the inner product of a real separable Hilbert space $H$ and $\|\cdot\|$ the associated norms in $H$ and $L(H, H)$. Instead of studying only the equation (3.3) we shall consider an equation of the form

$$
A z \equiv(I+\Lambda) z=b
$$

on the Hilbert space $H$. Here we assume that $\Lambda$ is a selfadjoint, compact operator on $H$ such that $A=I+\Lambda$ is positive definite and invertible.

The conjugate gradient method is an iterative method for the equation (4.1) which generates a sequence $\left\{z_{n}\right\}$ of approximations of the solution $z$ from an arbitrary initial approximation $z_{0}$. If $e_{n}=z-z_{n}$ and $r_{n}=A e_{n}$, then the method is given 
by

$$
\left\{\begin{array}{l}
z_{n+1}=z_{n}+\alpha_{n} s_{n}, \quad \alpha_{n}=\left\|r_{n}\right\|^{2} /\left(s_{n}, A s_{n}\right), \\
s_{n+1}=r_{n+1}+\beta_{n} s_{n}, \quad s_{0}=r_{0}, \quad \beta_{n}=\left\|r_{n+1}\right\|^{2} /\left\|r_{n}\right\|^{2}
\end{array}\right.
$$

For a survey of results for the conjugate gradient method we refer to Patterson [7]. For example, it is well known that the method is linearly convergent when it is applied to the equation (4.1). This result is obtained by only assuming that $A$ is uniformly positive definite on $H$. However, as it was observed by the author in [13] that, when $\Lambda$ is compact, the conjugate gradient method is $R$-superlinearly convergent (in the sense of Ortega and Rhineboldt [6]); i.e.,

$$
\left\|e_{n}\right\| \leqslant\left(c_{n}\right)^{n}\left\|e_{0}\right\|, \quad \text { where } \lim _{n \rightarrow \infty} c_{n}=0 .
$$

The following theorem was proved in [13].

THEOREM 4.1. Let $\Lambda$ be a selfadjoint, compact operator on $H$ such that $A=$ $I+\Lambda$ is positive definite. If $\left\{z_{n}\right\}$ is generated by (4.2), then $\left\{z_{n}\right\}$ converges $R$-superlinearly to the solution $z$ of (4.1).

If $\Lambda$ is restricted to a certain trace class, then it is also possible to give a convergence rate for the sequence $\left\{c_{n}\right\}$ in (4.3). For any $p \in[1, \infty)$ we let $C_{p}$ denote the von Neumann-Schatten class of operators on $H$. For details we refer, for example, to [9]. Here recall that the operator $\Lambda \in C_{p}$ if and only if $\Sigma_{j=1}^{\infty}\left|\lambda_{j}\right|^{p}<\infty$, where $\left\{\lambda_{j}\right\}$ is the spectrum of $\Lambda$. Furthermore, the associated $C_{p}$-norm is given by

$$
\|\| \Lambda \|_{p}=\left(\sum_{j=1}^{\infty}\left|\lambda_{j}\right|^{p}\right)^{1 / p}
$$

The theorem below, again proved in [13], shows that, if $\Lambda \in C_{p}$ for some $p \in[1, \infty)$, then $c_{n} \sim(1 / n)^{1 / p}$ as $n \rightarrow \infty$.

THEOREM 4.2. Let $A, \Lambda$ and $\left\{z_{n}\right\}$ be as in Theorem 4.1 and assume in addition that $\Lambda \in C_{p}$ for some $p \in[1, \infty)$. Then

$$
\left\|r_{n}\right\| \leqslant\left(\|A\|\left\|A^{-1}\right\|\right)^{1 / 2}\left(2(1 / n)^{1 / p}\left\|A^{-1}\right\|\|\Lambda \Lambda\|_{p}\right)^{n}\left\|r_{0}\right\| .
$$

We now return to the equation (3.3); i.e., $H=L^{2}(\Omega)$ and $\Lambda=R$. Since we have already seen that $R$ is a compact, selfadjoint operator on $L^{2}(\Omega)$, it follows that Theorem 4.1 applies to this equation. In order to see that Theorem 4.2 applies, we have to show that $R \in C_{p}$ for some $p \in[1, \infty)$. But this follows from a result of Pietsch and Triebel [8] which states that $L\left(L^{2}(\Omega), H^{q}(\Omega)\right) \subset C_{p}$ with continuous injection if $q>0$ and $1 \leqslant p<\infty$ are such that $p>d / q$. Hence, since $R \in$ $L\left(L^{2}(\Omega), H^{1}(\Omega)\right), R \in C_{p}$ for any $p>d$.

5. Problems with Positive Controls. We recall that in Section 3 we showed that the control problem (1.2) with $K=L^{2}(\Sigma)$ is equivalent to minimizing a quadratic functional over $L^{2}(\Omega)$. Throughout this section we consider (1.2) with $K=\{g \in$ $L^{2}(\Sigma) \mid g \geqslant 0$ a.e. on $\Sigma$ \}. We shall show that also in this case (1.2) is equivalent to 
minimizing a functional over the entire space $L^{2}(\Omega)$. Hence we transform the problem (1.2) into a minimization problem with no constraints on the space $L^{2}(\Omega)$. In this case the associated functional is not quadratic, but it is still strictly convex; and therefore, the minimization problem can be solved easily by iterative methods. In analogy with Section 3 two linear parabolic initial value problems have to be solved for each evaluation of the associated functional on $L^{2}(\Omega)$. We also observe that a direct discretization of (1.2) in the case of positive controls leads to a quadratic programming problem. Such problems are usually expensive to solve on a computer.

Now let $P_{K}$ denote the nonlinear projection of $L^{2}(\Sigma)$ onto $K$; i.e., $\left(P_{K} g\right)(\sigma)=$ $\sup (g(\sigma), 0)$. As in Section 3 we first consider the parabolic system which characterizes the optimal solution of (1.2). The following lemma is a consequence of the analysis given in [4] (see p. 124).

LEMMA 5.1. The control problem (1.2) has a unique solution. Furthermore, if $g \in K$ and $u=u_{g}(t, x)$, then $g$ is an optimal solution of (1.2) if and only if there is $a w \in W(0, T)$ such that

$$
\left\{\begin{array}{l}
(d u / d t, \varphi)+B(u, \varphi)=(f, \varphi)+\alpha^{-1}\left\langle P_{K}(-w), \varphi\right\rangle \text { for } \varphi \in H^{1}(\Omega) \\
-(d w / d t, \varphi)+B(\varphi, w)=0 \quad \text { for } \varphi \in H^{1}(\Omega), \\
u(0, \cdot)=v_{1}, \quad w(T, \cdot)=u(T, \cdot)-v_{2},
\end{array}\right.
$$

where $g=\alpha^{-1} P_{K}(-w)$.

Again we observe that the essential unknown in (5.1) is $w(T)$, in the sense that if $w(T)$ were known, then the solution can be found by solving parabolic initial value problems. In order to characterize $w(T)$, we define a nonlinear operator analog to the operator $R$ defined in Section 3. For any given $z \in L^{2}(\Omega)$ let $\hat{u}, \hat{w} \in W(0, T)$ be the solution of the system

$$
\left\{\begin{array}{l}
(d \hat{u} / d t, \varphi)+B(\hat{u}, \varphi)=-\alpha^{-1}\left\langle P_{K}(-\hat{w}), \varphi\right\rangle \quad \text { for } \varphi \in H^{1}(\Omega), \\
-(d \hat{w} / d t, \varphi)+B(\varphi, \hat{w})=0 \quad \text { for } \varphi \in H^{1}(\Omega) \\
\hat{u}(0, \cdot)=0, \quad \hat{w}(T, \cdot)=z,
\end{array}\right.
$$

and define a map $F: L^{2}(\Omega) \rightarrow L^{2}(\Omega)$ by $F(z)=\hat{u}(T)$.

We note that, if $\hat{u}, \hat{w}$ are given by (5.2), then $\hat{w}(t)=E(T-t) z$, where $E(t)$ was defined in Section 2. For any $\varphi \in L^{2}(\Omega)$ we now have

$-\alpha^{-1}\left\langle P_{K}(-\hat{w}), E(T-t) \varphi\right\rangle=\left(\frac{d \hat{u}}{d t}, E(T-t) \varphi\right)+B(\hat{u}, E(T-t) \varphi)=\frac{d}{d t}(\hat{u}, E(T-t) \varphi)$.

Hence we see by integration that

$$
(F(z), \varphi)=-\alpha^{-1} \int_{0}^{T}\left\langle P_{K}(-E(t) z), E(t) \varphi\right\rangle d t .
$$


In particular, if $z_{1}, z_{2} \in L^{2}(\Omega)$, then

$$
\begin{aligned}
\left(F\left(z_{1}\right)-\right. & \left.F\left(z_{2}\right), z_{1}-z_{2}\right) \\
& =-\alpha^{-1} \int_{0}^{T}\left\langle P_{K}^{\prime}\left(-E(t) z_{1}\right)-P_{K}\left(-E(t) z_{2}\right), E(t)\left(z_{1}-z_{2}\right)\right\rangle d t \geqslant 0
\end{aligned}
$$

Hence, $F$ is a monotone operator on $L^{2}(\Omega)$. Furthermore, we note that since $P_{K}$ is contractive it follows from the regularity of the map (2.2) and from (5.3) that there is a constant $M>0$ such that

$$
\begin{aligned}
\left\|F\left(z_{1}\right)-F\left(z_{2}\right)\right\| & \leqslant \sup _{\|\varphi\|=1} \alpha^{-1} \int_{0}^{T}\left|E(t)\left(z_{1}-z_{2}\right)\right||E(t) \varphi| d t \\
& \leqslant M\left\|z_{1}-z_{2}\right\| .
\end{aligned}
$$

We now note that, if $u, w$ is the solution of (5.1) and $z^{*}=w(T)$, then

$$
z^{*}+v_{2}=u(T)=u_{0}(T)-F\left(z^{*}\right) \quad \text { or } \quad z^{*}+F\left(z^{*}\right)=b_{0},
$$

where $b_{0}=u_{0}(T)-v_{2}$.

We also observe that since $F$ is monotone and Lipschitz continuous it follows, for example, from Vainberg [10] that (5.6) has a unique solution in $L^{2}(\Omega)$. In fact, if we define a sequence of approximations $\left\{z_{n}\right\}_{n=0}^{\infty}$ of $z^{*}$ by $z_{n+1}=z_{n}-\epsilon G\left(z_{n}\right)$, $z_{0}$ arbitrary, where $G(z)=z+F(z)-b_{0}$ then $\left\{z_{n}\right\}$ converges to $z^{*}$ for $0<\epsilon<$ $2(1+M)^{-2}$. However, this is not a practical numerical method since a sharp bound for the Lipschitz constant $M$ is hard to obtain.

In order to obtain a more practical iterative method for (5.6), we first show that $G$ is a potential operator; i.e., $G$ is the gradient of a nonlinear functional on $L^{2}(\Omega)$. First observe that $F$ is a positive homogeneous map; i.e.,

$$
F(\beta z)=\beta F(z) \quad \text { for any } \beta \geqslant 0 .
$$

Hence, the only possible functional (up to addition of a constant) is given by

$$
\Psi(z)=\int_{0}^{1}(G(\theta z), z) d \theta=\frac{1}{2}(z+F(z), z)-\left(b_{0}, z\right) .
$$

THEOREM 5.1. Let $\Psi$ be the nonlinear functional on $L^{2}(\Omega)$ defined above. Then $\Psi$ is Frechet differentiable everywhere in $L^{2}(\Omega)$ and $\nabla \Psi(z)=G(z)$.

Proof. We first note that since every continuous Gâteaux derivative is a Fréchet derivative (see, for example, [10]), it is enough to show that $G$ is the Gâteaux derivative of $\Psi$. In order to show this, it is enough to show that $F$ is the Gateaux derivative of

$$
\widetilde{\Psi}(z) \equiv \frac{1}{2}(F(z), z)=\frac{1}{2 \alpha} \int_{0}^{T}\left|E^{-}(t) z\right|^{2} d t,
$$

where $\left(E^{-}(t) z\right)(x)=\inf (0,(E(t) z)(x))$. Recall that it follows from [5] that, if $\sigma \in \Sigma$ is fixed and if $\left\{\varphi_{n}\right\}$ converges to $\varphi$ in $L^{2}(\Omega)$, then $\left\{\left(E(\cdot) \varphi_{n}\right)(\sigma)\right\}$ converges to $(E(\cdot) \varphi)(\sigma)$. 
Now let $z, h \in L^{2}(\Omega), \beta \in \mathbf{R}, \beta \neq 0$, and consider

$$
\begin{aligned}
\omega(\beta) & \equiv \frac{1}{\beta}[\tilde{\Psi}(z+\beta h)-\widetilde{\Psi}(z)]-(F(z), h) \\
& =\frac{1}{2 \beta}[(F(z+\beta h), \beta h)+(F(z+\beta h)-F(z), z)]-(F(z), h) \\
& =\frac{1}{2 \alpha} \int_{0}^{T}\left\langle E^{-}(t)(z+\beta h)-E^{-}(t) z, E(t) h\right\rangle d t \\
& +\frac{1}{2 \alpha} \int_{0}^{T}\left[\frac{1}{\beta}\left\langle E^{-}(t)(z+\beta h)-E^{-}(t) z, E(t) z\right\rangle-\left\langle E^{-}(t) z, E(t) h\right\rangle\right] d t .
\end{aligned}
$$

Hence, since $\lim _{\beta \rightarrow 0} \omega(\beta)=0$ by the dominated convergence theorem, $F$ is the Gâteaux derivative of $\tilde{\Psi}$.

We now observe that, if $z^{*}$ is the solution of (5.6), then $z^{*}$ minimizes the strictly convex functional $\Psi$ over $L^{2}(\Omega)$. In order to find the minimum of this functional we can now apply any reasonable method for minimizing a strictly convex functional. We shall not go into a detailed study of algorithms for minimizing $\Psi$ here, but we should like to mention a version of the steepest descent method which is globally linearly convergent and where no a priori bounds need be known.

Consider the method

$$
z_{n+1}=z_{n}-\lambda_{n} G\left(z_{n}\right), \quad z_{0} \text { arbitrary, }
$$

where $\lambda_{n}$ is given by

$$
\lambda_{n}=\frac{\left\|G\left(z_{n}\right)\right\|^{2}}{\left((I+R) G\left(z_{n}\right), G\left(z_{n}\right)\right)} .
$$

Here $R$ is the linear operator defined by (3.2). We note that in the same way as we derived (5.3) it follows for $z, \varphi \in L^{2}(\Omega)$ that

$$
(R z, \varphi)=\alpha^{-1} \int_{0}^{T}\langle E(t) z, E(t) \varphi\rangle d t
$$

Hence, it follows from (5.4) that

$$
0 \leqslant(F(z)-F(\varphi), z-\varphi) \leqslant(R(z-\varphi), z-\varphi) .
$$

By using (5.9) and the mean value theorem it now follows that, if $\left\{z_{n}\right\}$ is generated by (5.7) and (5.8), then

$$
\Psi\left(z_{n+1}\right) \leqslant \Psi\left(z_{n}\right)-\frac{\left\|G\left(z_{n}\right)\right\|^{4}}{2\left((I+R) G\left(z_{n}\right), G\left(z_{n}\right)\right)} .
$$

Even if the choice of $\lambda_{n}$ given by (5.8) is not the best possible, the estimate (5.10) can be used as a test in order to guarantee fast convergence of a method of the form (5.7).

Finally, we should like to consider the possibility of obtaining superlinearly convergent iterations for the equation (5.6). These methods could for example be certain 
quasi-Newton methods (see the survey paper [1] by Dennis and More). Since a necessary condition for obtaining superlinear convergence is that $F$ be continuously Fréchet differentiable, we shall give a condition that implies that $F$ has this property.

We assume that for any $z \in L^{2}(\Omega), z \neq 0$,

$$
m(\{(t, \mu) \in \Sigma \mid(E(t) z)(\mu)=0\})=0,
$$

where $m(\cdot)$ denotes the surface measure on $\Sigma$. A sufficient condition for (5.11) to be satisfied is that $\partial \Omega$ be analytic and that the coefficients $a_{i, j}$ and $c$ of $L$ be analytic (see for example $[4$, p. 125]).

For any $z \in L^{2}(\Omega)$ define $T_{z} \in L\left(L^{2}(\Omega), L^{2}(\Omega)\right)$ by

$$
\left(T_{z} \xi, \varphi\right)=\alpha^{-1} \int_{0}^{T}\left\langle E_{z}(t) \xi, E(t) \varphi\right\rangle d t
$$

for any $\xi, \varphi \in L^{2}(\Omega)$, where

$$
\left(E_{z}(t) \xi\right)(x)= \begin{cases}(E(t) \xi)(x), & \text { if }(E(t) z)(x)<0 \\ 0, & \text { otherwise. }\end{cases}
$$

We note that $T_{z}$ is selfadjoint since $\left\langle E_{z}(t) \xi, E(t) \varphi\right\rangle=\left\langle E_{z}(t) \xi, E_{z}(t) \varphi\right\rangle$. Also, $E_{z}(t) z$ $=E^{-}(t) z$.

ThEOREM 5.2. Assume that the condition (5.11) is satisfied. Then $F$ is continuously Fréchet differentiable for all $z \in L^{2}(\Omega), z \neq 0$, and $F^{\prime}(z)=T_{z}$.

Proof. We first recall from the theory of analytic semigroups (see, for example, [3] ) that there is a constant $c_{2}>0$ such that for any $z \in L^{2}(\Omega)$

$$
\|E(t) z\|_{1} \leqslant c_{2} t^{-1 / 2}\|z\|, \quad 0<t \leqslant T,
$$

where $\|\cdot\|_{1}$ denotes the norm on $H^{1}(\Omega)$. It also follows from [5] that there is a constant $c_{3}>0$ such that for any $\varphi \in H^{1}(\Omega)$,

$$
|\varphi| \leqslant c_{3}\|\varphi\|_{1}^{1 / 2}\|\varphi\|^{1 / 2} \text {. }
$$

Hence, for any $z \in L^{2}(\Omega)$

$$
|E(t) z| \leqslant c_{4} t^{-1 / 4}\|z\|, \quad 0<t \leqslant T,
$$

where $c_{4}>0$ is independent of $z$.

Now let $z \in L^{2}(\Omega), z \neq 0$, be given and for any $h \in L^{2}(\Omega)$ consider

$$
\omega(h)=F(z+h)-F(z)-T_{z} h .
$$

We would like to show that $\lim _{\|h\| \rightarrow 0}\|\omega(h)\| /\|h\|=0$. In order to do so let first

$$
\beta(h)=\left.\alpha^{-1}\left(E^{-}(t)(z+h)-E^{-}(t) z-E_{z}(t) h\right)\right|_{\Sigma} .
$$

Then for any $\varphi \in L^{2}(\Omega)$,

$$
(\omega(h), \varphi)=\int_{0}^{T}\langle\beta(h), E(t) \varphi\rangle d t .
$$

Now let $\epsilon>0$ be given. First note that, since

$$
\langle\beta(h), E(t) \varphi\rangle \leqslant 2 \alpha^{-1}|E(t) h||E(t) \varphi|,
$$


it follows from (5.12) that there exist a $\rho=\rho(\epsilon), 0<\rho \leqslant T$, such that

$$
\int_{0}^{\rho}\langle\beta(h), E(t) \varphi\rangle d t \leqslant \frac{\epsilon}{2}\|h\|\|\varphi\| .
$$

Define now for each $\delta>0$

$$
S_{\delta}=\left\{(t, \mu) \in \Sigma_{\rho}||(E(t) z)(\mu) \mid \leqslant \delta\right\},
$$

where $\Sigma_{\rho}=\{(t, \mu) \in \Sigma \mid t \geqslant \rho\}$. We note that condition (5.11) implies that $\lim _{\delta \rightarrow 0} m\left(S_{\delta}\right)=0$. Hence, there is a $\delta=\delta(\epsilon)>0$ such that

$$
\int_{S_{\delta}} \beta(h) E(t) \varphi d \sigma \leqslant \frac{\epsilon}{2}\|h\|\|\varphi\|
$$

Finally, for each $h \in L^{2}(\Omega)$ let

$$
\widetilde{S}_{\delta, h}=\left\{(t, \mu) \in \Sigma_{\rho}||(E(t)(z+h))(\mu)-(E(t) z)(\mu) \mid \geqslant \delta\right\} .
$$

Now note that it follows from (5.13), (5.14) and from the definition of $\beta(h)$ that for any $h, \varphi \in L^{2}(\Omega)$

$$
|(\omega(h), \varphi)| \leqslant \epsilon\|h\|\|\varphi\|+\int_{\widetilde{S}_{\delta, h}}|\beta(h) E(t) \varphi| d \sigma .
$$

But since the map $\left.h \rightarrow E(\cdot) h\right|_{\Sigma_{\rho}}$ is a continuous map from $L^{2}(\Omega)$ into $C\left(\Sigma_{\rho}\right)$ it follows that there is a constant $\gamma>0$ such that

$$
\widetilde{S}_{\delta, h}=\varnothing \quad \text { for }\|h\| \leqslant \gamma .
$$

Hence, $\lim _{\|h\| \rightarrow 0}\|\omega(h)\| /\|h\|=0$, which implies that $F$ is Fréchet differentiable at $z$ and that $F^{\prime}(z)=T_{z}$. In order to see that $F^{\prime}(z)$ is continuous we have to use an argument similar to the one above. This will be omitted here.

As a final observation we note that the techniques in this section can also be applied to elliptic control problems. Numerical methods for such problems were, for example, studied by Falk in [2]. Also, in this case we obtain numerical methods that seem to be more efficient than those provided by solving a quadratic programming problem.

\footnotetext{
Department of Mathematics

University of Chicago

Chicago, Illinois 60637
}

1. J. E. DENNIS \& J. MORÉ, "Quasi-Newton methods, motivation and theory," SIAM Rev., v. 19,1977 , pp. 46-89.

2. R. S. FALK, "Approximation of a class of optimal control problems with order of convergence estimates," J. Math. Anal. Appl., v. 44, 1973, pp. 28-47.

3. A. FRIEDMAN, Partial Differential Equations, Holt, Rinehart and Winston, New York, 1969.

4. J. L. LIONS, Optimal Control of Systems Governed by Partial Differential Equations, Springer-Verlag, New York, 1971.

5. J. L. LIONS \& E. MAGENES, Non Homogeneous Boundary Value Problems and Applications, Vols. I-II, Springer-Verlag, New York, 1972.

6. J. M. ORTEGA \& W. C. RHEINBOLDT, Iterative Solution of Nonlinear Equations in Several Variables, Academic Press, New York, 1970. 
7. W. M. PATTERSON, Iterative Methods for the Solution of a Linear Operator Equation in Hilbert Space, Lecture Notes in Math. vol. 394, Springer-Verlag, New York, 1974.

8. A. PIETSCH \& H. TRIEBEL, "Interpolationstheorie für Banachideale von beschränkten linearen Operatoren," Studia Math., v. 3, 1968, pp. 95-109.

9. J. R. RINGROSE, Compact Non-Self-Adjoint Operators, Van Nostrand Reinhold Co., London, 1971.

10. M. M. VAINBERG, Variational Method and Method of Monotone Operators in the Theory of Nonlinear Equations, Wiley, New York, 1973.

11. R. WINTHER, A Numerical Galerkin Method for a Parabolic Control Problem, Ph.D. Thesis, Cornell University, 1977.

12. R. WINTHER, "Error estimates for a Galerkin approximation of a parabolic control problem," Ann. Mat. Pura Appl. (4), v. 107, 1978, pp. 173-206.

13. R. WINTHER, "Some superlinear convergence results for the conjugate gradient method," SIAM J. Numer. Anal. (To appear.) 